INRA, Prod. Anim., 2005, 18 (2), 79-86

\title{
Les croisements franco-américains chez le trotteur : une expérience réussie?
}

A. RICARD

INRA, Station de Génétique Quantitative et Appliquée, F-78352 Jouy-en-Josas Cedex Courriel : aricard@germinal.toulouse.inra.fr

Faut-il continuer à utiliser Workaholic, le célèbre trotteur américain étalon national longtemps stationné au Haras du Pin et seul représentant 100 \% étranger faisant encore la monte en France ? En se croisant avec le Trotteur Américain (le Standardbred), le Trotteur Français y a-t-il laissé son âme ? Ou au contraire peut-on parler de réussite pour ce protocole de croisement, limité dans le temps mais dont l’influence est aujourd'hui grandissante ?

La population de trotteurs français a connu, dès la fin du 19e siècle et dans les quarante premières années du 20e siècle, un apport constant et significatif de gènes standardbred. Après la fermeture du StudBook en 1937, le Trotteur Français (TF) est resté fermé sur lui-même pendant plusieurs décennies (à l'exception des croisements avec le Pur Sang) alors que le Trotteur Américain, le Standardbred, se répandait un peu partout en Europe. Dans les années 80 , on a commencé à ouvrir la porte à l’apport de gènes étrangers. Commencée par petites touches (utilisation des 1/2 américains Florestan et Granit (H.N.), importation d'un étalon national américain Mickey Vicking, mort prématurément et remplacé rapidement par Workaholic, autorisation de saillie à l'étranger pour une poignée de juments, achat de quelques poulinières standardbred), la diffusion des gènes américains dans le Trotteur Français est aujourd'hui généralisée. Quelle est l’influence de ce croisement sur les performances en course du Trotteur Français? Quel est le taux actuel de gènes étrangers ? Qui $a$ été et est aujourd'hui responsable de sa diffusion?

\section{1 / Données généalogiques et données de performances}

Le fichier généalogique des Trotteurs Français est tenu par les Haras

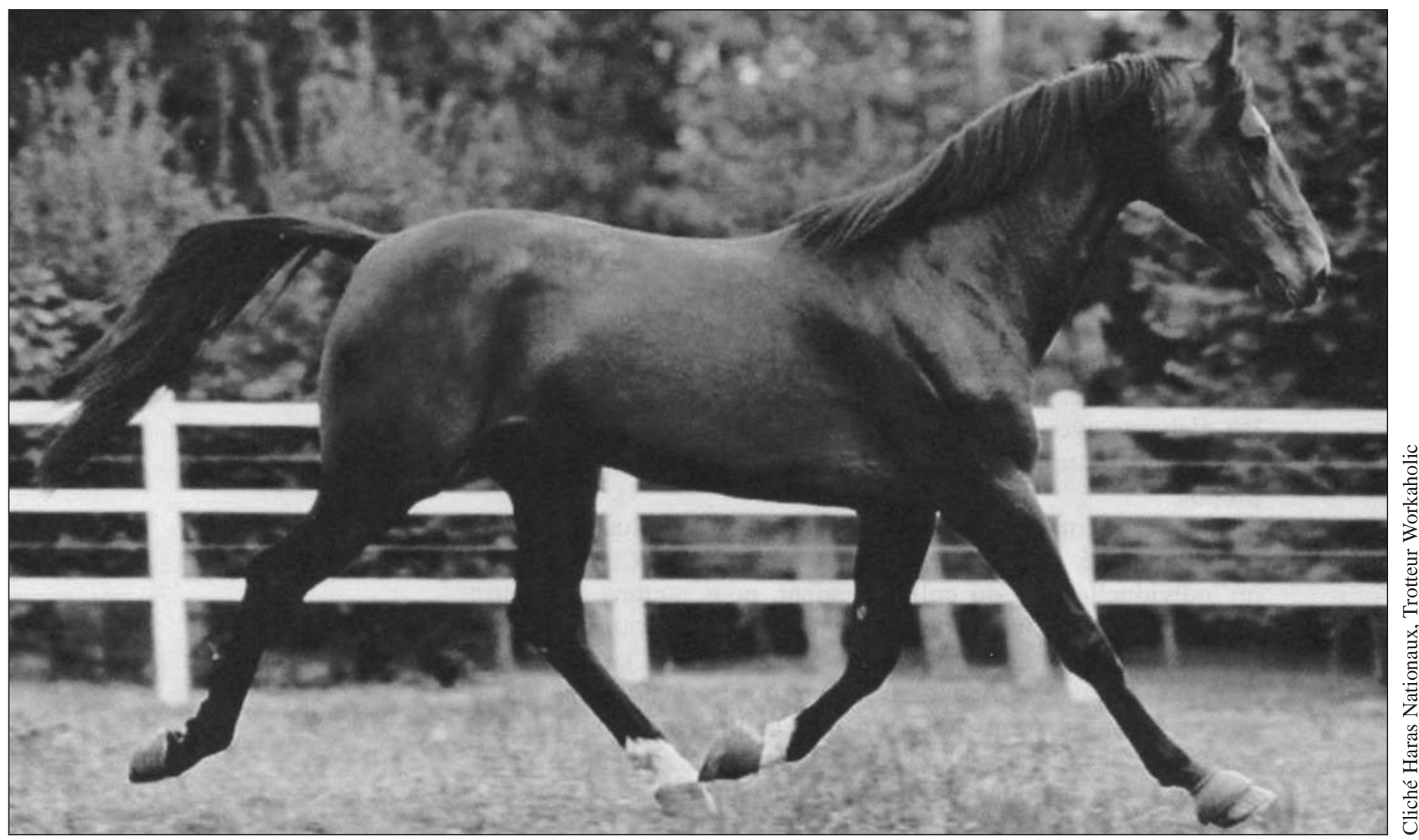


Nationaux grâce au SIRE (Système d'Identification Répertoriant les Equidés). Les naissances sont enregistrées de façon exhaustive depuis 1974 et les généalogies sont remontées antérieurement grâce aux documents d'accompagnement des premiers animaux enregistrés.

\section{1 / Fichier de base}

Pour étudier les origines des trotteurs français, nous avons extrait tous les chevaux de race Trotteur Français et Trotteur Etranger (TE, facteur ou non de Trotteur Français, c'est-à-dire pouvant ou non produire des TF) inscrits au SIRE et tous leurs ancêtres, quelle que soit leur race. On a ainsi de façon exhaustive l'ensemble des naissances Trotteur Français depuis 1974 jusqu'en 2001 et leurs ancêtres sur le nombre de générations utilisées lors de l'inscription au SIRE. Pour donner une idée de la profondeur de pedigree que cela représente, on peut noter que pour les TF nés récemment, entre 1995 et 1999 , on remonte de façon quasi exhaustive 5 générations (98\% des ancêtres connus), et généralement 6 générations (84 \% des ancêtres connus). Cinq générations correspondent à 32 parents fondateurs (c'est-à-dire eux-mêmes sans parents connus) et 62 ancêtres (chevaux présents dans le pedigree), six générations à 64 parents fondateurs et 126 ancêtres.

Le fichier total représente 305104 chevaux différents. Les informations disponibles sont :

- le numéro d'identification du cheval ;

- le numéro d’identification du père ;

- le numéro d’identification de la mère ;

- la race. Pour les trotteurs, seule est distinguée l'appellation Trotteur Français de l'appellation Trotteur Etranger, sans distinction précise de race parmi les races étrangères de trotteurs. Il existe aussi des trotteurs étrangers facteurs de trotteurs français (TE(TF)). Les autres ancêtres possibles sont les Pur Sang (PS) ;

- la date de naissance ;

- le pays de naissance ;

- l'indice annuel de performance pour chaque année de participation aux courses (ITR) ;

- la somme des gains à 5 ans (cumul jusqu'en 2004, donc complet pour les naissances avant 2000).

L'indice annuel de performance (ITR) est le logarithme du gain annuel divisé par le nombre de sorties en cour- se dans l'année corrigé pour les effets de l'âge et du sexe et standardisé chaque année à une moyenne de 100 et un écart-type de 20. C'est la mesure de base qui sert à l'indexation génétique (Langlois 1989, Tavernier 1989), il mesure la réussite précoce (à 3 ans) comme un taux de rentabilité par rapport au nombre de sorties réalisées. La somme des gains de 2 ans à 5 ans est utilisée comme un critère de réussite globale sur la carrière du cheval. Pour obtenir une distribution proche de la normalité, une transformation de BoxCox a été utilisée avec une puissance de 0,15 . Ce critère de performance est corrigé pour les effets de sexe et d'année de naissance et standardisé à un écart type de 20 .

\section{2 / Définition de la contribu- tion des reproducteurs à la population actuelle / définition des ancêtres importants}

Il est possible de déterminer l'espérance du nombre de copies d'un gène d'un ancêtre à un locus donné dans la population des trotteurs nés aujourd'hui quelle que soit la place de l'ancêtre dans le pedigree. Ce sont les calculs de probabilité d'origine de gènes (Vu Tien Khang 1983). On calcule de proche en proche la contribution de l'ancêtre grâce à cette règle simple :

soit $n$ le nombre de chevaux de la population actuelle de référence (par exemple la population née une année donnée).

Le père $i$ de $z$ produits dans cette population est responsable de :

$$
P_{i}=\frac{Z}{2 n} \text { gènes de la génération }
$$

Le père $j$ de ce père sera lui même responsable de :

$$
P j=\frac{P_{i}}{2}=\frac{Z}{4 n} \quad \begin{aligned}
& \text { gènes de la généra- } \\
& \text { tion actuelle }
\end{aligned}
$$

et ainsi de suite en remontant le pedigree jusqu'aux parents «originels», ceux qui n'ont eux-même pas de parents connus. Pour un même ancêtre, on somme évidemment toutes ses contributions possibles par toutes ses branches descendantes possibles.

Pour l'analyse, utiliser tous les contributeurs est redondant. Utiliser uniquement les fondateurs évite la redondance mais ne met pas en évidence d'éventuels goulots d'étranglements intermédiaires entre la génération d'aujourd'hui et celle des ancê- tres fondateurs. Nous préférerons donc la notion d'ancêtre important. Seuls les ancêtres qui contribuent le plus à la génération d'aujourd'hui vont être retenus mais en s'arrangeant pour supprimer le problème des redondances.

Un ancêtre important se définit par le pourcentage de gènes qu'il a légué à la population de référence (Boichard et al 1996 et 1997). On calcule ce pourcentage pour tous les ancêtres. On choisit l'ancêtre qui a contribué le plus (qui a le plus grand pourcentage) et on considère que c'est le premier ancêtre important. On supprime alors pour tous ses propres ancêtres et ses produits (sur toutes les générations possibles) leur contribution à la génération d'aujourd'hui qui a transité par cet ancêtre important. On recalcule alors pour tous les ancêtres moins l'ancêtre le plus important, leur nouvelle contribution à la génération de référence. On a alors la contribution de chacun moins celle qui a éventuellement transité par l'ancêtre le plus important. On choisit alors le cheval qui a, à nouveau, contribué le plus et on considère que c'est le deuxième ancêtre le plus important. On procède ainsi jusqu'à obtenir $100 \%$ des contributions ou bien le nombre d'ancêtres importants qu'on désire. Cette liste des ancêtres importants est plus pertinente que celle qu'on peut faire à partir de la contribution de tous les ancêtres fondateurs et est non redondante : le nième ancêtre important est bien celui qui contribue le plus sans compter sa contribution éventuelle par les ancêtres classés avant lui. Une programmation propre a été utilisée, notamment pour adapter la méthode à l'étude de la contribution d'une seule race dans une autre.

\section{3 / Règles d'appellation de race}

Les règles de désignation de la race d'un produit en fonction de celles de ses parents ont varié dans le temps compte tenu des règlements en vigueur aux différentes époques. Il n'y a donc pas de règle unique pour attribuer la race d'un croisement TF et TE. Pour estimer au mieux le pourcentage que chaque race représente dans le patrimoine génétique d’un trotteur, il est nécessaire de remonter les origines. La règle retenue est la suivante. Un cheval sans parents connu est $100 \%$ de la race dans laquelle il est déclaré. On détermine ensuite le pourcentage de gènes origi- 
naires des différentes races pour le produit de ces chevaux sans parents selon :

$\%$ de race $z$ du produit $=1 / 2$ (\% race $z$ du père $+\%$ race $z$ de la mère).

Et on procède ensuite de la même manière pour les produits de ce cheval et ainsi de suite jusqu'aux dernières générations nées. Les pourcentages de chaque race sont donc entièrement déterminés par la race des chevaux fondateurs, c'est-à-dire dont on ne remonte pas le pedigree, indépendamment des appellations utilisées ensuite au cours des générations. Ainsi Mickey Vicking, bien que qualifié de TF dans le fichier SIRE est bien considéré dans l'étude comme ayant 100 \% de race étrangère.

\section{4 / Contribution ancienne et récente des gènes étrangers}

L’apport de gènes étrangers dans le Trotteur Français s'est faite en deux temps : d'une part à la création du TF, par l'utilisation d'étalons américains avant la deuxième guerre mondiale (Sam Williams, The Great MC Kinney, Net Worth...) et d'autre part de façon récente par la reproduction franco-américains (Florestan, Granit (H.N.), Kimberland), l'achat d'étalons américains (Mickey Viking, Workaholic), la mise en place du protocole franco-américain de saillie de juments TF par des étalons TE et l'achat de juments TE. Il est intéressant de distinguer dans l'étude ces deux apports (avant/ après guerre) qui ne se sont pas fait en même temps et pas pour les mêmes raisons.

La totalité de l'apport récent de gènes TE s'est faite par l'intermédiaire de :

- 20 étalons (nés entre 1947 et 1985) : Armbro Goal, Ayres, Baltic Speed, Bonefish, Mack The Knife, Meadow Road, Mickey Vicking, Napoletano, Nevele Pride, Pershing, Prakas, Quick Pay, Quick Song (pas directement mais via son fils italien Sharif Di Iesolo, issu d'une mère TF), Royal Prestige, Speedy Crown, Speedy Somolli, Star's Pride, Sugarcane Hanover, Super Bowl, Workaholic ;

- 22 poulinières (nées entre 1975 et 1988), importées en France et ayant eu des produits TF : Armbro Elegant, Armbro Glamour, Bermuda Star, Camille Lobell, Castleton Class, Fleur Lobell, Floral Sea, Honey Rroasted, Grades Gaiety, Marlena Lobell, Mary Lobell, Mayday Lobell, No No
Nannette, Perfect Mate, Proximity Lane, Sedation, Seven Palms, Singoalla, Shemein, Snow, Tableau, Velvet Slipper.

On a donc attribué à ces chevaux et leurs ancêtres la race TE. Tous les autres TE, et donc notamment les anciens étalons (Sam Williams, The Great Mc Kinney, The Harvester, Maxworthy...) sont considérés comme TF.

Grâce à ces simples modifications, tous les gènes TE d'avant guerre se voient transformés en TF et l'étude porte bien sur l'apport de gènes $\mathrm{TE}$ récent. C’est cette importation récente que nous étudions ici, qui concerne des chevaux sélectionnés de façon intensive aux Etats-Unis sur la course mais dans des conditions différentes des conditions françaises : l'accent est mis sur la précocité (chevaux aptes à 2-3 ans) et sur des hippodromes très standardisés (pistes plates) par rapport aux hippodromes français (nature du terrain, montées/descentes). C'est une étude différente de celle de l'effet du $\mathrm{TE}$ en tant que fondateur (entre autres) du TF au moment de sa création au début du $19^{\mathrm{e}}$ siècle.

\section{2 / Résultats}

\section{1 / Evolution de l'apport des gènes Trotteur Etranger}

La population TF née en 2001 (11070 chevaux) a 0,9 \% de gènes PS (Pur Sang), 88,5 \% de gènes TF et $10,7 \%$ de gènes TE. Si l'apport de PS n’a quasiment pas varié depuis 1974 (passant de 1,1\% à 0,9\%), celui de TE, depuis sa première introduction dans les naissances de 1978 a considérablement augmenté comme le montre la figure 1 .

On a nettement quatre phases d'évolution. La première apparition date de 1978 et le taux de gènes $\mathrm{TE}$ demeure confidentiel jusqu'en 1984 (0,2 \%). La deuxième phase est une augmentation régulière assez faible et s’étale de 1984 à 1993 avec une augmentation de 0,3 \% par an. On passe ainsi de 0,2 \% de gènes TE en 1984 à 2,6 \% en 1993, en 9 ans. On a ensuite une accélération notable sur 3 ans : de 1993 à 1996 avec une augmentation de 1,5 point de pourcentage par an. On arrive ainsi en 1996 à un taux moyen de $7 \%$ de gènes TE. On a maintenant une augmentation régulière et assez forte, même si elle est plus modérée que sur la période 1993/ 1996. L'augmentation est maintenant de 0,75 point de pourcentage par an. Ces quatre phases correspondent à 4 phases différentes dans l'introduction et la multiplication des gènes étrangers.

Cette augmentation est due à l'augmentation du nombre de chevaux TF ayant des gènes TE par rapport à l'ensemble des naissances $\mathrm{TF}$ et non à l'augmentation du taux de TE pour chaque cheval porteur de gènes étrangers (figure 2). En 2001, les deux tiers de la population TF a des gènes TE ! Mais le taux moyen de gènes $\mathrm{TE}$ de ceux qui en sont porteurs est assez stable depuis plus de 10 ans (1988) et tourne autour de $15 \%$ (un peu

Figure 1. Evolution du pourcentage de gènes TE dans la population TF née depuis 1974 en fonction de l'année de naissance.

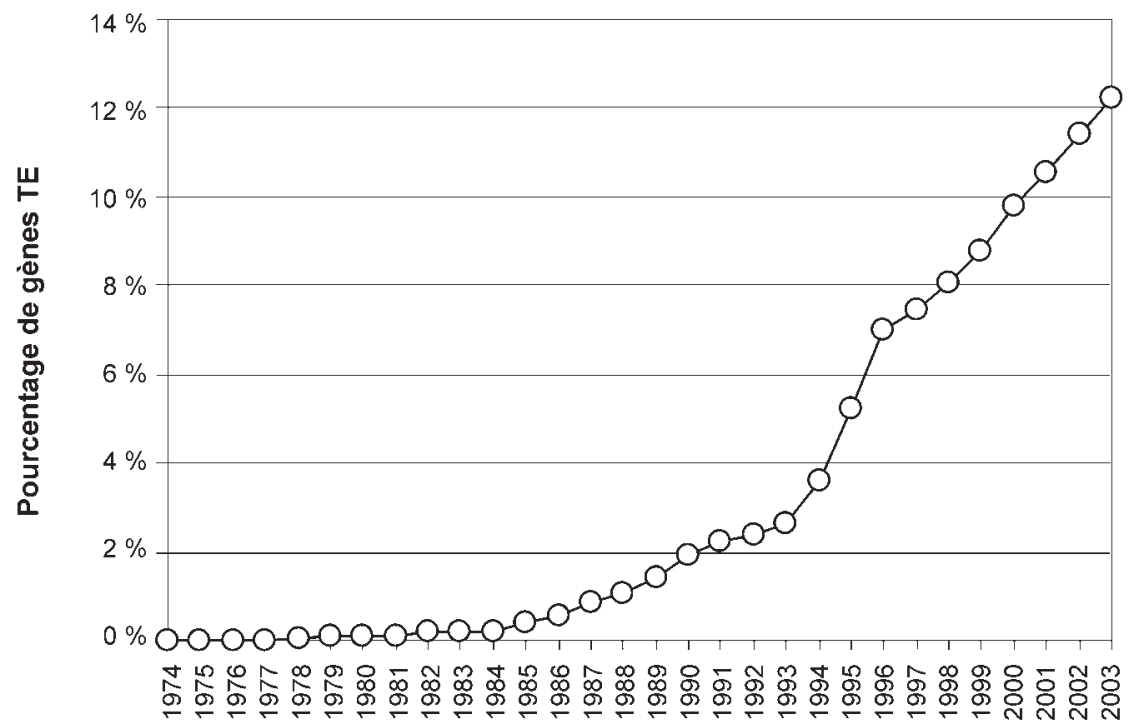

Année de naissance 
Figure 2. Evolution de la proportion de la population de TF ayant des gènes TE et de la moyenne du pourcentage de gènes TE des chevaux porteurs en fonction de l'année de naissance.

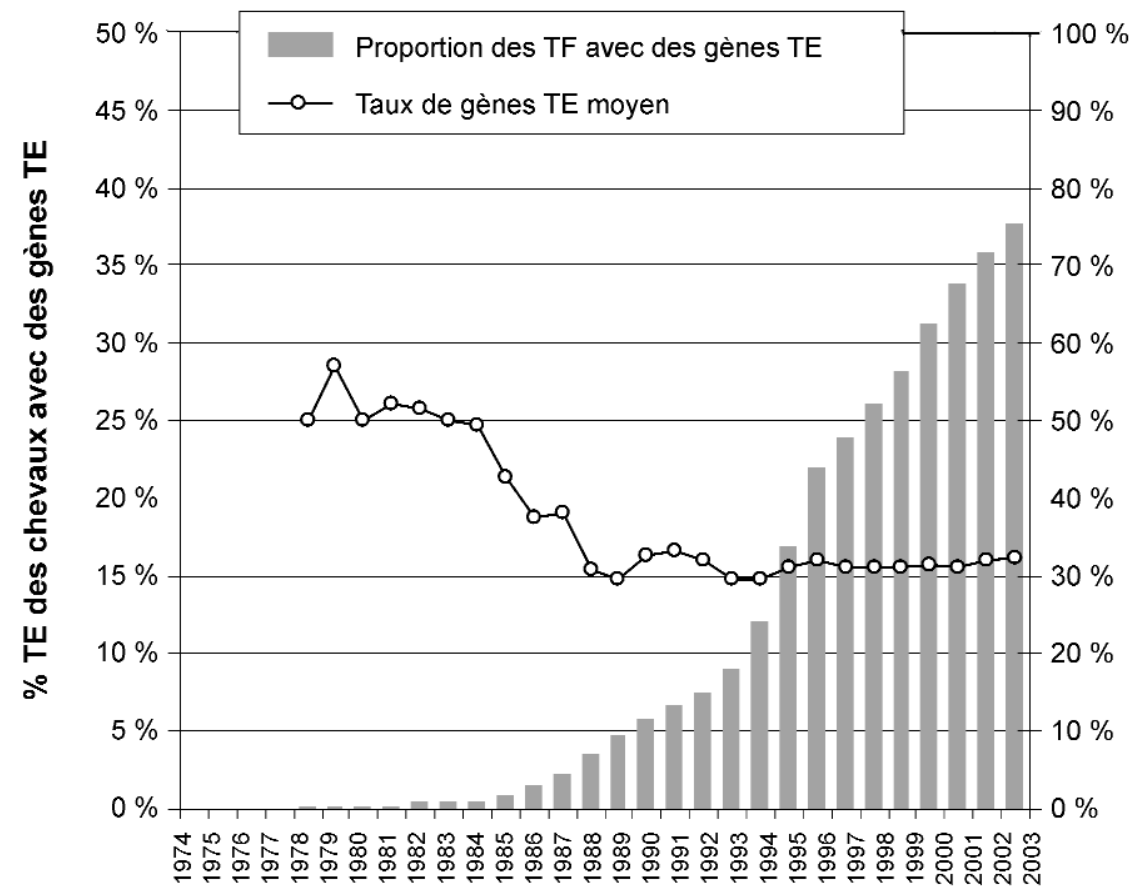

Année de naissance

au dessus de $1 / 8^{\mathrm{e}}$ de gènes $\mathrm{TE}$, soit équivalent à un arrière grandpère TE).

\section{2 / Mode d'introduction des gènes Trotteur Etranger}

Les gènes TE sont introduits d'une part par les mâles, d'autre part par les femelles. Dans un premier temps c'est la reproduction d'étalons ayant des gènes TE $(100 \%, 1 / 2,1 / 4 .$.$) qui va$ créer l'augmentation du nombre de chevaux ayant des gènes TE dans la population mais aujourd'hui $20 \%$ des gènes $T E$ ont pour origine les juments issues des premiers croisements (figure 3 ).

Du coté des mâles, deux phénomènes vont jouer : d'une part l'augmentation du nombre d'étalons TF ayant des gènes TE mis à la reproduction, d'autre part l'augmentation du nombre de saillies de ces étalons par rapport aux étalons $100 \%$ TF.

Le nombre d'étalons porteurs de gènes TE n'a cessé d'augmenter depuis le début de leur introduction, d'abord modérément (on passe de 11 étalons en 1986 à 40 en 1991) puis fortement (111 en 1996, 204 en 2001). L'augmentation aujourd'hui régulière est d'un peu moins de 20 nouveaux TE 50 ! gènes Trotteur Etranger.

\section{3 / Contribution des étalons ayant des gènes Trotteur Etranger à leur diffusion}

La figure 3 représente la contribution relative des différentes classes de reproducteurs à la diffusion totale des gènes TE par année de naissance. Comme reproducteurs, on distingue les femelles des mâles et parmi les mâles, on classe les étalons selon leur propre taux de gènes $\mathrm{TE}$.

On distingue 3 phases si on se situe à partir de 1988, année où le taux de TE atteint $1 \%$ dans la population TF. Dans un premier temps, ce sont les étalons $1 / 4$ de gènes $\mathrm{TE}$ qui sont responsables de la diffusion des gènes TE dans la population, puis paraissent les $1 / 2$ de gènes TE qui en 3 ans (de 1994 à 1996) dépassent en importance les 1/4 de gènes et assurent aujourd'hui $50 \%$ de la diffusion des gènes TE, de façon stable depuis ces 6 dernières années. Les 1/4 TE ne représentent aujourd'hui qu'un peu plus de $38 \%$ de la diffusion des gènes $\mathrm{TE}$, ils sont maintenant secondés par les 1/8 et les 1/16 de gènes TE qui, malgré un faible taux de gènes TE pour l'étalon, représentent quand même $12 \%$ de la diffusion. Le rôle des 100 \% TE, s’il a représenté dans la fin des années 80 jusqu'à $20 \%$ de la diffusion, est aujourd'hui à la marge (moins de $5 \%$ ).

La diffusion des gènes $\mathrm{TE}$ est de plus en plus l'affaire d'un nombre important d'étalons, le rôle de chaque étalon devient de plus en plus anecdotique face à l'ensemble des contributions. On peut calculer le nombre

Figure 3. Part relative des différentes catégories de reproducteurs dans la diffusion des

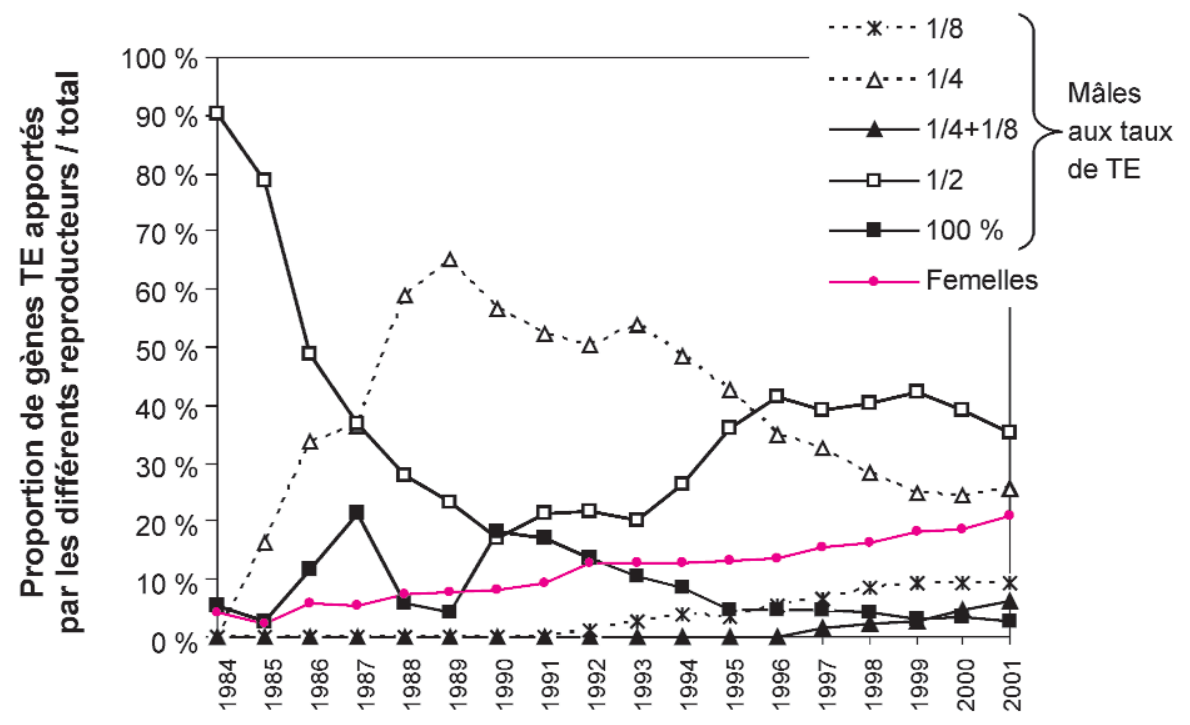

Année de naissance 
minimum d'étalons nécessaire pour obtenir un pourcentage donné de gènes TE par rapport au total de gènes TE. Ainsi, si pendant la période 19851993, 10 étalons minimum étaient responsables de $50 \%$ de la diffusion des gènes étrangers par les mâles, il en faut 21 pour la période 1994-1996 et 31 aujourd'hui. Pour obtenir $80 \%$ des gènes TE, il fallait 25 étalons sur la période 1985-1993, 50 sur la période 1994-1996 et 81 sur la période 1997-2001.

\section{4 / Distribution du taux de Trotteur Etranger dans la popu- lation actuelle de référence}

Nous nous intéressons particulièrement aux chevaux nés entre 1995 et 1999 qui sont ceux sur lesquels nous allons tester l'influence sur les performances (de façon à avoir un recul suffisant et une possibilité de sortie en course à l'âge de 3 ans). Sur les 59079 naissances TF de 1995 à 1999 (figure 4), il y a près de la moitié $(46,8 \%)$ de TF qui ont des gènes TE. La plus grande partie (40\%) de ces chevaux sont des 1/8 de gènes (donc par exemple avec un arrière grand-père TE). Il y a environ un quart de chevaux avec 1/16 de gènes TE (soit équivalent à un arrière-arrière grand-père), et un quart avec 1/4 de gènes TE (soit avec un grand-père TE). L'introduction de gènes TE commence à être assez lointaine et la diffusion de ces gènes se fait à partir d'animaux croisés, avec un faible taux de
Figure 5. Effet du \% de gènes Trotteur Etranger sur l'ITR (indice de performance basé sur le gain par départ) à 3 ans et un indice normalisé basé sur la somme totale des gains à 5 ans, ( \pm 1 écart type résiduel) - Chevaux TF nés entre 1995 et 1999.

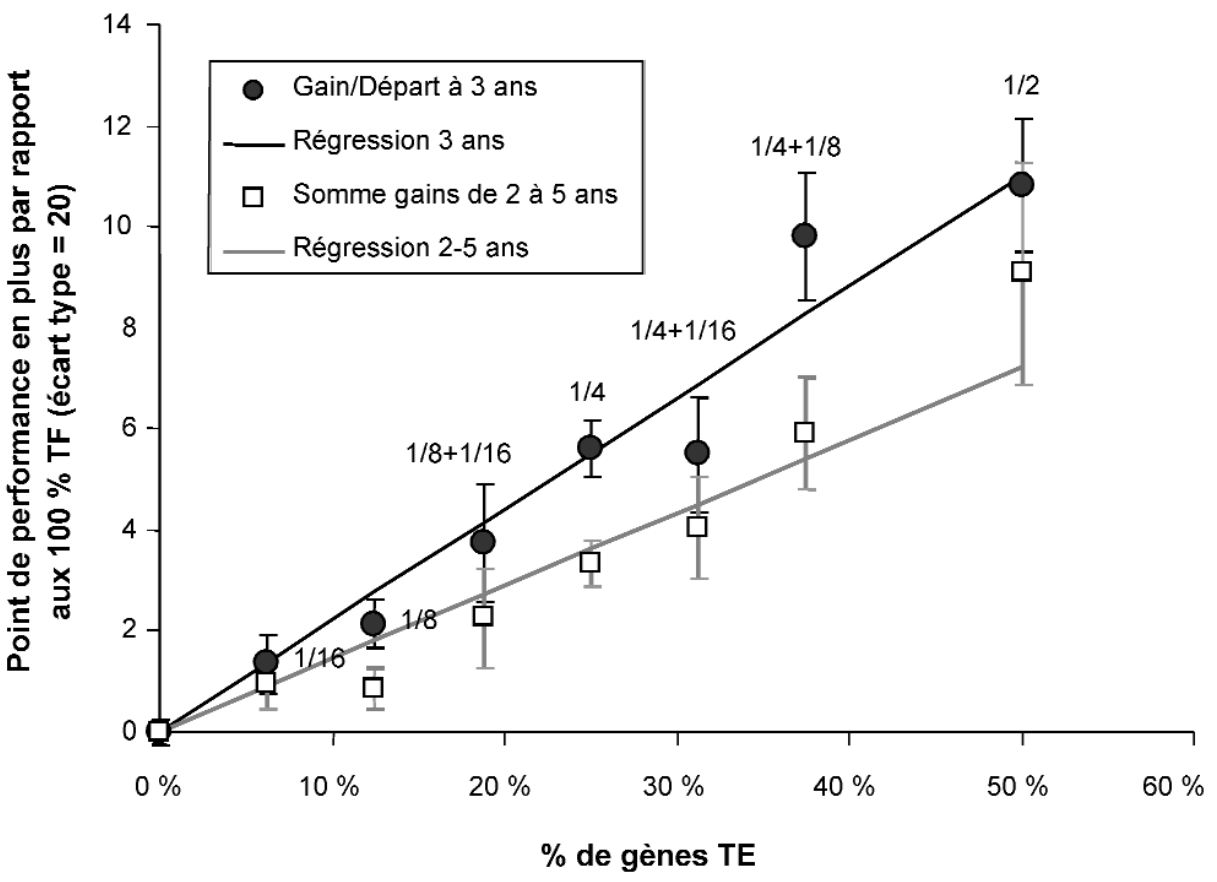

gènes TE, mais largement répandus dans la population.

\section{5 / Influence du taux de gènes Trotteur Etranger sur les per- formances}

On a mesuré l'influence du taux de gènes TE sur la performance par son

Figure 4. Distribution de la proportion de gènes TE chez les TF nés de 1995 à 1999 ayant des gènes TE.

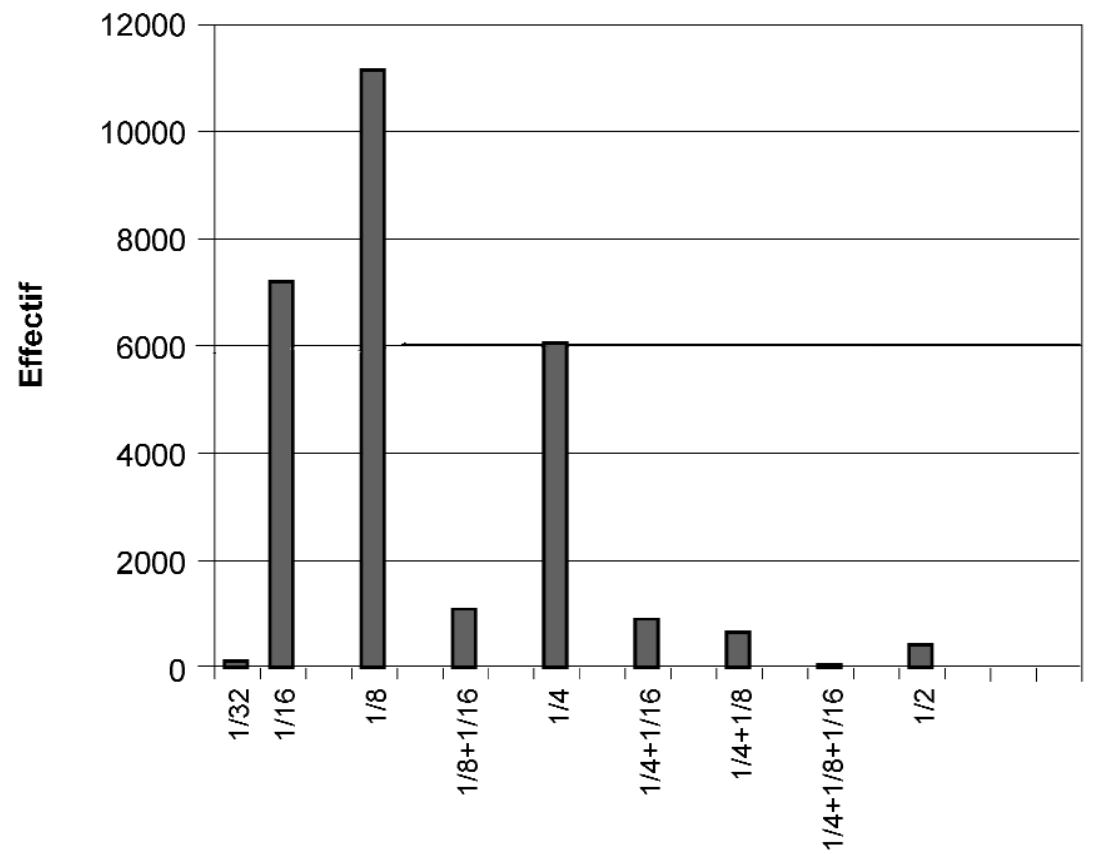

Taux de gènes TE influence sur l'ITR obtenu à 3 ans et la somme des gains jusqu'à 5 ans transformée (figure 5). La figure donne l'estimation des moindres carrés de l'effet sur la performance des chevaux regroupés selon des puissances de $1 / 2$ et le résultat de la régression. Dans la population des TF nés de 1995 à 1999, l'influence des gènes TE sur la performance en course est favorable et régulière en fonction du taux de gènes TE. On gagne 2,2 points d'indice par tranche de $10 \%$ de gènes TE en plus pour l'ITR à 3 ans et 1,4 point d'indice pour le gain total cumulé à 5 ans. De plus, $25 \%$ des chevaux TF nés avec des gènes $\mathrm{TE}$ sont gagnants en course à 3 ans contre $17 \%$ des $100 \% \mathrm{TF}$, et $34 \%$ entre 2 et 5 ans contre $24 \%$ des purs TF. Cependant la régression des ITR sur le taux de TE n'explique que 1,7\% de la variabilité à 3 ans et $0,7 \%$ sur le cumul à 5 ans, même si elle est hautement significative $(\mathrm{p}<0,0001)$.

\section{3 / Discussion}

\section{1 / Analyse de la diffusion des gènes Trotteur Etranger}

Nous avons vu que nous avions quatre phases dans la diffusion des gènes TE dans la population TF. La première période de 1978 à 1984 de très faible introduction de gènes étrangers correspond aux premières saillies des 
1/2 américains (mères françaises) Florestan et Granit (H.N.). A eux deux, ils représentent 93,5 \% des gènes TE diffusés mais cela ne concerne que $0,6 \%$ des naissances. La deuxième période de 1985 à 1993 d'augmentation modérée correspond au début du pur américain Workaholic mais il ne représente que $9 \%$ de la diffusion des gènes TE. Les 1/2 américains Florestan et Granit (H.N.), auxquels s’ajoute maintenant Kimberland représentent $19 \%$ de la diffusion. Ce sont finalement les 1/4 TE (principalement des fils de Florestan), qui sont majoritaires dans la diffusion des gènes TE (52 \%). L'apport des juments augmente et passe à 9,8\% du total. L'accélération de la diffusion des gènes TE vient de l'augmentation du nombre d'étalons avec des gènes TE : 4,8 \% des étalons en service mais surtout du nombre de saillies par étalon plus élevé : 13,3 produits par an contre 6,8 pour les étalons n’ayant pas de gènes TE.

La troisième période de croissance la plus importante des gènes $\mathrm{TE}$ entre 1994 et 1996 correspond à une explosion dans l'utilisation des étalons ayant des gènes TE. Le nombre d'étalons ayant des gènes TE passe de 72 à 115, et le nombre moyen de produits par an passe de 13,3 à 29,7. Plus on avance dans le temps et plus la diffusion des gènes $\mathrm{TE}$ se répartit sur un nombre important d'étalons, il n'y a plus vraiment d'étalon «marquant» : Workaholic est toujours le plus important mais ne représente plus que 5,6 \% des gènes TE diffusés totaux. En revanche on commence à voir apparaître ses fils (6 fils pour 6,0 \%) et les fils des croisements des juments françaises du protocole franco-américain. Ce sont donc les 1/2 TE qui deviennent prépondérants (36,1 \%). Les chevaux issus de parents ayant des gènes TE représentent maintenant 33,6 \% des naissances. La voie femelle est responsable de 13,2 \% de la diffusion des gènes TE.

La quatrième période de croissance, toujours importante mais plus modérée que les trois années passées s'étend de 1997 à 2001. Le nombre de produits par étalon ayant des gènes TE se stabilise (24 par an en moyenne sur la période) mais leur nombre va toujours croissant (235 étalons, soit 24,5 \% des étalons en service). Désormais près d' $1 / 5$ de la diffusion des gènes TE est assurée par les juments (18 \%). En ce qui concerne les étalons, on a à la fois une augmentation de l'influence des 1/2 TE ( $47 \%$ des gènes TE diffusés par la voie mâle contre $42 \%$ avant) et une augmentation du rôle des 1/8 TE (11 \% contre $5 \%$ ) à la défaveur des $1 / 4 \mathrm{TE}$ (33\% contre $47 \%$ ).

\section{2 / Ancêtres importants}

La part de gènes TE actuelle (naissance 1995 à 1999) est expliquée par seulement 32 chevaux TE différents. Seuls ces 32 chevaux ont laissé des gènes dans la population d'aujourd'hui, aucun autre gène TE ne vient d'ailleurs, et $99 \%$ des gènes TE d'aujourd'hui sont expliqués par seulement 13 chevaux différents, 95 \% par 9 chevaux.

La figure 6 illustre les croisements entre les ancêtres majeurs responsables de la diffusion des gènes TE. Ce graphique explique à lui seul $99 \%$ des gènes étrangers dans le TF né de 1995 à 1999. Les trois principaux fondateurs du TE d'aujourd'hui sont Star's Pride (46,4\%), Speedy Crown (18,5\%) et Hoot Mon (8,8 \%). Mais ces lignées ne sont pas distinctes et s'entrecroisent pour donner tous les grands étalons ayant du sang TE utilisés directement en France.

Figure 6. Ancêtres responsables de 99 \% des gènes TE dans la population TF née de 1995 à 1999.

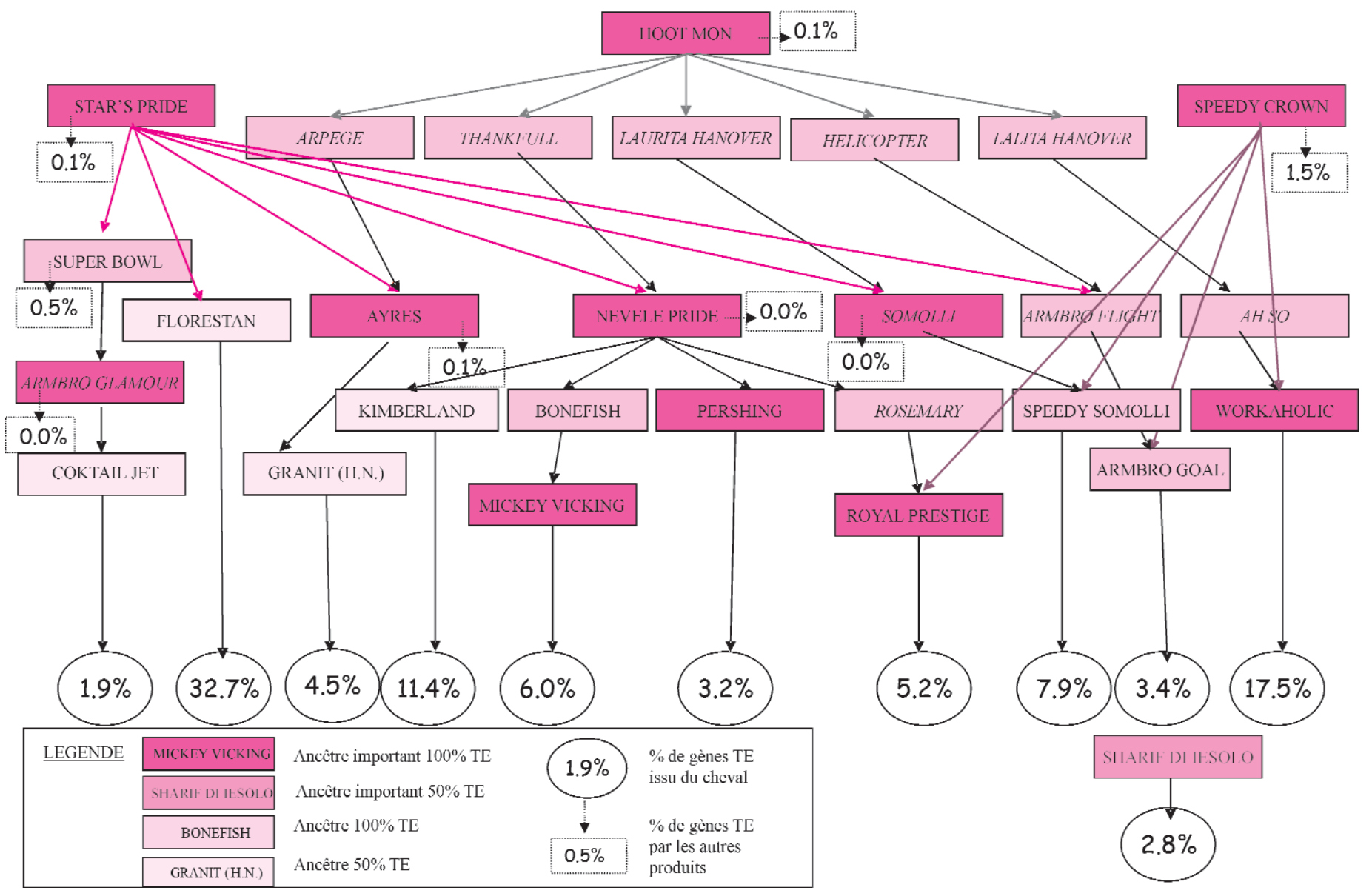




\section{3 / Influence du taux de gènes Trotteur Etranger sur les per- formances}

Le calcul n'a pas pour ambition de déterminer l'écart de qualité entre le Trotteur Français et le Trotteur Etranger. Il mesure uniquement l'influence de ces croisements, tels qu'ils ont été pratiqués, avec les étalons qu'on a utilisés, sur les performances des produits. Le fait que ce résultat soit positif ne veut pas dire qu'on aurait obtenu le même résultat avec n'importe quels étalons américains ou qu'on obtiendrait à nouveau le même résultat en important d'autres étalons. D'autre part, il n'est pas possible non plus par ce simple calcul de faire la part des choses entre l'influence strictement génétique et la part environnementale : il y a fort à parier que les produits issus des étalons TE ou 1/2 TE ont bénéficié, au moins au début de l'expérience, d'un environnement largement plus favorable que leurs contemporains $100 \%$ TF. Cependant il semblerait malgré tout que l'avantage perdure même pour les $1 / 8$ et $1 / 16$ de gènes américains, donc avec une introduction qui commence à être plus lointaine et avec sans doute moins d'effets environnementaux particuliers. L'effet est favorable mais demeure modeste : 2,2 points pour $10 \%$ de gènes $\mathrm{TE}$, cela veut dire moins de 6 points de mieux pour les 1/4 TE. L'effet est plus marqué sur des performances précoces (3 ans), et sur un critère qui mesure la réussite rapportée au nombre de départs, que sur les performances cumulées à l'âge adulte sur un critère qui met plus l'accent sur la longévité. A 5 ans, les 1/4 TE n’ont plus finalement que 3 points d'avance sur les $100 \% \mathrm{TF}$.

\section{4 / Assiste-t-on à une «holstei- nisation» du Trotteur Français comme de la race bovine Française Frison-Pie Noire dans les années 80 ?}

Croiser une race française avec une race étrangère dont la supériorité génétique est évidente est un moyen rapide de progresser dans une aptitude donnée. C’est ce qu’ont réalisé les éleveurs de vaches Frisonnes partout en Europe de l'Ouest avec les Holstein nord-américaines dans les années 1980. Ces croisements ont amené rapidement à une absorption complète d'une race par l'autre : en 1990, les taureaux étaient quasiment à $100 \%$ Holstein et les femelles avaient plus de $83 \%$ de gènes Holstein alors que ce taux était proche de 0 dans le début des années 70 (Boichard et al 1993a et 1993b). Nous sommes en fait dans une situation totalement différente. La diffusion des gènes Holstein s'est faite par les taureaux d'importation de race pure Holstein qui ont été régulièrement utilisés jusqu'à l'absorption complète. Chez le Trotteur Français, nous l'avons vu, très peu d'étalons 100 \% TE ont été utilisés en France, la diffusion s'est surtout faite par des 1/2 TE puis des 1/4 TE sans qu'il y ait retour sur la race parentale TE (Back Cross) et donc sans augmentation du taux de gènes TE autre que par la plus grande diffusion des croisés au sein de la population par rapport aux étalons purs TF. C'est ce qui explique la généralisation de la présence des gènes $\mathrm{TE}$ dans toute la population TF mais son taux stable et encore faible de $15 \%$. Cela explique aussi l’évolution parallèle des 1/4, 1/8 et 1/16 TE dans la population alors que les Françaises Frisons Pie Noire sont rapidement passés des 1/2 aux 3/4 puis 7/8.

\section{5 / Influence sur l'indexation, estimation des paramètres géné- tiques du croisement}

Dans l'étude de Boichard et al (1993a, 1993b), il avait été possible de calculer les paramètres génétiques du croisement : les différences additives entre lignées, les effets d’hétérosis et de perte de recombinaison et d'en tirer des conséquences sur l'évaluation génétique qui négligeait les paramètres spécifiques du croisement (hétérosis, perte de recombinaison). Cette étude est impossible ici compte tenu même du type d'introduction des gènes TE. En effet, on a vu qu'on remontait sur très peu d'ancêtres TE, or ces ancêtres, si on les introduit dans une évaluation génétique de type modèle animal prenant en compte toutes les parentés, ne sont représentés en France que par leurs produits étalons exceptionnels et non par l'ensemble de leur production. Chez les bovins laitiers, les taureaux importés étaient testés sur descendance et la base de comparaison était donc possible. Ici une évaluation génétique classique conduit à surestimer considérablement les valeurs génétiques des Purs américains ancestraux et donc leurs produits. A tel point qu'il a fallu rompre certaines chaînes de parenté pour donner des évaluations génétiques correctes. Ainsi les étalons TE ayant des performances propres à l'étranger et ayant moins de 15 produits nés en France avec des performances en course ne sont pas estimés par un modèle animal. Ils sont estimés uniquement par leur propre performance grâce à une correspondance avec une somme de gains française. Ils interviennent dans l'estimation de leurs produits comme un père différent pour chaque produit avec cette performance équivalente. L'information apportée par les $1 / 2$ frères n'est donc pas utilisée pour les produits de ces étalons. De plus, l'estimation des paramètres spécifiques du croisement aurait été difficile compte tenu du faible nombre de 1/2 TE porteurs de tout l'hétérosis et de la rapide diminution de cet effet pour les générations suivantes.

\section{Conclusion}

L'apport de gènes TE à la population TF touchera bientôt toutes les naissances (les 2/3 aujourd'hui). Il y a un contraste entre la diffusion actuellement très large des gènes TE à partir d'un grand nombre d'étalons $1 / 2$ ou $1 / 4$ TE et même des poulinières $1 / 8$ et la concentration des origines TE utilisées au moment de l'introduction puisque 13 ancêtres seulement expliquent $99 \%$ des gènes TE actuels. La période du croisement, et donc de l'aventure Workaholic, est déjà dans l'histoire, la diffusion se fait aujourd'hui par les reproducteurs croisés. L'effet «brut» de l'apport de gènes TE semble positif sur la performance en course à 3 ans mais n'explique qu'une très petite fraction des différences de performance entre chevaux (2\%) et est moins marqué sur l'ensemble de la carrière à 5 ans. Il n'est pas possible de déterminer si cet effet est principalement génétique ou environnemental, les traitements préférentiels suivant les mêmes voies que la génétique. Mais la pérennité de cet effet sur les chevaux à faible taux TE $(1 / 8,1 / 16)$, la linéarité de l'influence en fonction du pourcentage de gènes TE pourrait faire pencher la balance vers la génétique. 

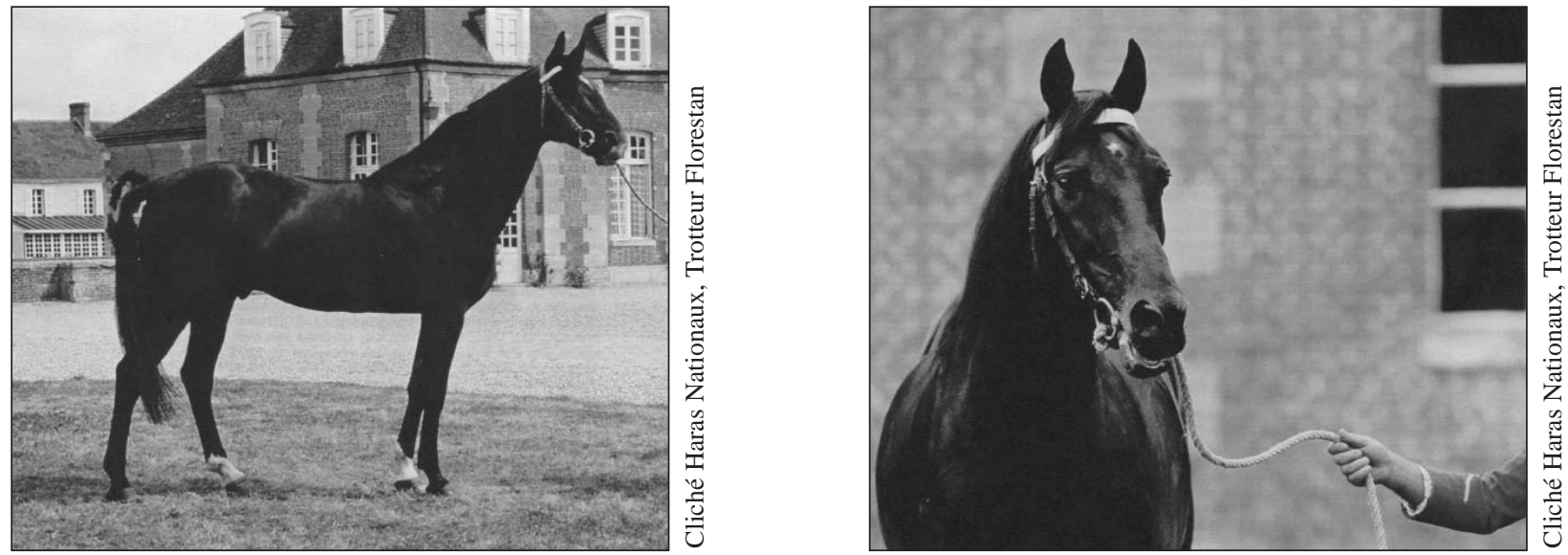

\section{Références}

Boichard D, Bonaiti B, Barbart A, 1993a. Effet du croisement Holstein sur les caractères laitiers en population Pie Noire. INRA Prod. Anim., 6, 25-30.

Boichard D., Bonaiti B. Barbat A., 1993b. Effect of Holstein crossbreeding in the French black and white cattle population. J. Dairy Sci., 76, 1157-1162.

Boichard D., Maignel L., Verrier, E., 1996. Analyse généalogique des races bovines laitières françaises. INRA Prod. Anim., 9, 323-335.
Boichard D., Maignel L., Verrier, E., 1997. The value of using probabilities of gene origin to measure genetic variability in a population. Genet. Sel. Evol., 29, 5-23.

Langlois B., 1989. Breeding evaluation of French trotters according to their race earnings. 1. Present situation. In : EAAP publication (ed.) State of breeding evaluation in trotters. Pudoc, Wageningen, 27-40.

Tavernier A., 1989. Caractérisation de la population Trotteur Français d'après leur estima- tion génétique par un BLUP modèle animal. Ann. Zootech., 38, 145-155.

Tavernier A., 1989. Breeding evaluation of French trotters according to their race earnings. 2. Prospects. In : EAAP publication (ed.) State of breeding evaluation in trotters. Pudoc, Wageningen, 95-105.

Vu Tien Khang J., 1983. Méthodes d'analyse des données démographiques et généalogiques dans les populations d'animaux domestiques. Genet. Sel. Evol., 15, 263-298.

\section{Résumé}

Les 3/4 des Trotteurs Français nés en 2003 ont des gènes étrangers issus des croisements réalisés avec le Standardbred américain depuis 1978. La part de gènes américains de ces chevaux est de $16 \%$, ce qui donne une proportion de $12 \%$ de gènes américains pour l'ensemble de la population Trotteur Français. La diffusion des gènes américains s’est réalisée principalement par le 1/2 américain Florestan et ses produits (33\%). L’apport du pur américain Workaholic (et ses produits) ne représente que $17 \%$. C’est une introduction très différente de la holsteinisation de la race bovine Pie Noire car il n'y a pas de retour actuellement sur la race parentale étrangère et le taux de gènes étrangers ne s'est maintenu que par l'utilisation préférentielle des étalons porteurs de gènes étrangers par rapport aux purs français (31 produits annuels contre 13). Maintenant que l'introduction est achevée, le taux de gènes étrangers est stabilisé et généralisé, il perdure par accouplement des animaux croisés. Cette large diffusion contraste avec le faible nombre de fondateurs américains : 13 ancêtres suffisent à expliquer $99 \%$ des gènes américains dans les Trotteurs Français ! L’influence du taux de gènes étrangers sur la performance (indice standardisé à un écart-type de 20) est favorable et linéaire. Elle est plus importante sur le gain par départ à l'âge de 3 ans (2,2 points à 3 ans pour $10 \%$ de gènes étrangers) qu’à 5 ans sur le gain total cumulé de la carrière (1,4 point) mais n'explique que $2 \%$ de la variabilité.

\section{Abstract}

Standardbred crossbreeding in the French trotter horse population: A successful experiment?

The percentage of French trotters born in 2003 with Standardbred genes was $75 \%$. The mean value of the proportion of standardbred genes of these horses was $16 \%$ and so the average proportion of Standardbred genes in the whole population of French trotters was $12 \%$. The reason was mainly the use of the $1 / 2$ American stallion Florestan and its offspring (33\%). The influence of the American stallion Workaholic and its offspring was only $17 \%$. This crossbreeding was very different from the cross of the Holstein breed in Black and White dairy cattle during the 1980's. There was no back cross with the American population but with the French population and the percentage of Standardbred genes was maintained by the superior use of stallions with American genes (31 offspring per year versus 13). The introduction of Standardbred genes has been achieved and the percentage of genes was stable due to mating between crossed horses. The high percentage of French trotters with Standardbred genes contrasted with the small number of American founders: 13 ancestors were sufficient to explain $99 \%$ of the Standardbred genes! The effect of Standardbred genes on race performance was favourable and linear. The effect was greater for the annual earning per start at 3 years (11\% of phenotypic deviation for $10 \%$ of American genes) than for total cumulated life earning at 5 years of age (7\% of phenotypic deviation). In any case this effect explained less than $2 \%$ of the variability.

RICARD A., 2005. Les croisements franco-américains chez le trotteur : une expérience réussie ? INRA Prod. Anim., 18, 79-86. 